\title{
Preliminary study of weave pattern influence on microplastics from fabric laundering
}

Textile Research Journa 2021, Vol. $91(9-10)$ 1037-1045 (C) The Author(s) 2020 Article reuse guidelines: sagepub.com/journals-permissions DOI: $10.1177 / 0040517520965708$ journals.sagepub.com/home/trj

SAGE

\author{
María Berruezo, Marilés Bonet-Aracil (D), Ignacio Montava, \\ Eva Bou-Belda, Pablo Díaz-García $\mathbb{D}$ and Jaime Gisbert-Payá
}

\begin{abstract}
Nature, including the oceans, is polluted by the presence of plastics. Different products can be found, including plastic bottles, toys, toothpaste tubes, fruit meshes, etc. Small pieces of plastics, known as microplastics, have been found in the oceans and there is concern that their impact is increasing. Some of those microplastics are considered to come from the textile products. In this article, the authors will suggest how to minimize the environmental problem of the presence of microplastics in wastewaters from textile laundries. The aim of this study is to determine the influence of some parameters related to the design of fabrics. A relationship between microplastic release and parameters from weave design will be established. A fiberglass filter was used to analyze water from the laundry. Results demonstrated that the interlacing coefficient influences the number of particles in the wastewaters. Moreover, it was demonstrated that the higher the density of yarns/cm in the fabric, the lower the quantity of fibers could be found in the wastewater. Results demonstrated that the presence of weave patterns, such as a plain pattern, work better from the sustainability point of view than a twill. The interlacing coefficient and the weft density are important to prevent the microplastic release.
\end{abstract}

\section{Keywords}

textile, fabric, design, rapport, weave pattern, microplastic

Currently, plastic is essential in our way of life, and we can find it in the majority of everyday objects and in products of this material. ${ }^{1}$ Percentages of plastics allocated are as follows: $36 \%$ to containers and packaging; $16 \%$ to construction; $15 \%$ to textiles; $22 \%$ to consumer products and other fields; $6 \%$ to transport; $4 \%$ to electrical and electronic items; and $1 \%$ to industrial machinery. ${ }^{2}$

Plastics are synthetic polymers that are derived from oil or gas; the first plastic is known as bakelite. ${ }^{3}$ Since then, development in this field has increased so markedly that the production of plastics in the first 10 years of the 21st century has almost been matched the production throughout the middle of the last century. ${ }^{4}$

This has become a major environmental problem, since an overproduction of this matter has been achieved in most of the manufacturing of products of all types. This overproduction leads to misuse and disposal of these plastics, which is a very powerful source of environmental pollution.
The marine environment is one most affected by this problem, and is the most problematic. Beaches, surface waters, columns of sea currents and subtidal sediments are all affected by these petroleum derivatives, which can come from various sources, such as the fragmentation of larger plastics or the direct entry of microplastics derived from cosmetics or resulting particles from the wear of products. ${ }^{4}$ Some articles ${ }^{5}$ evaluate the water footprint of plastics, or design new applications of fibers in order to implement a circular economy. ${ }^{6}$ In addition to these sources, recently, the sources of

Departamento de Ingeniería Textil y Papelera, Escuela Politécnica Superior de Alcoy, Universitat Politècnica de València, Spain

\section{Corresponding author:}

Marilés Bonet-Aracil, Departamento de Ingeniería Textil y Papelera, Escuela Politécnica Superior de Alcoy, Universitat Politécnica de Valéncia, Plaza Ferrandiz y Carbonell s/n Alcoy, 0380I Spain. Email: maboar@txp.upv.es 
synthetic fibers derived from household washes were rated with a priority of $7 / 10$ degrees. ${ }^{1}$

In the fashion world there are many materials found on the market that use different types of weave patterns, densities and laminar mass. The variety of textile structures is endless, making an immense range of possibilities and qualities. Even so, the vast majority of textile products contain synthetic fibers. Currently, these types of fibers are commonly present in textile products, since due to their use, the qualities of the garments or products are improved. Some examples of improvement are the elasticity or the perspiration capacity in the textile structures.

The increase in discounts and rebates, together with the reduction of production terms, benefits this new style of consumption, so that in 2014 the production of 100 billion textile items was exceeded. Currently, this industry is working on moving in the direction of a circular economy, seeking to reduce the amount of textile waste, increase the number of uses of the product and recycle as much as possible. ${ }^{7}$

Data from studies conducted by Greenpeace say that in a few years the increase in clothing consumption will be $63 \% .{ }^{8}$ However, this trend may change as a result of increased awareness, partly derived from the involvement of social agents, for instance, politicians, media, etc.

Even so, the consumerism implanted in the current population derives from a high demand for fibers, which amounted to 99 million tons in 2016, with $62.7 \%$ being synthetic fibers. At this rate, a demand of 130 million tons of fibers is expected in 2025, implying an increase in cotton and polyester production of $40 \%{ }^{7}$

Consequently, the increase in textile waste is escalating, ${ }^{7}$ adding the environmental impact generated by synthetic fibers, releasing up to 1 million microplastic fibers in a single wash, according to Greenpeace. ${ }^{7}$ The microplastics themselves are not a problem, but they can become a serious problem for life around them. ${ }^{9,10}$ The problem with microplastics is how imperceptible they are. Even more, despite their reduced size, they can carry pollutants such as heavy metals. ${ }^{9}$ Thus, when research is conducted to find them, they appear even in domestic salt ${ }^{11}$ and in some fats. ${ }^{12}$

The attempt to change the sector is a very important challenge, since it supposes the revision of productive process in the search for the reduction of harmful chemical products, residual waters and damaging overproductions, and to implement new techniques that are respectful with the environment.

Greenpeace supports this change by offering campaigns such as the "Detox," which encourages the transformation of the textile industry, focusing on the elimination of hazardous chemicals, the review of production and the re-evaluation of the fiber production and wet processes. The main challenge is the reduction of waste caused by rapid fashion. Eighty companies committed to embarked on the "Detox" project, launched by Greenpeace, representing $15 \%$ of world clothing production. ${ }^{8}$

In spite of the progressive advance of the inclusion of the circular economy in textiles, the washing of garments is necessary, which implies the discharge of the fibers to rivers and marine waters. This release occurs in both industrial and household washes due to mechanical and chemical activity in the wash. The dimensions of expelled microplastics are not detected by wastewater treatment plants before the wastewater enters seas and oceans. ${ }^{13}$ This affects both fauna and marine flora, as the microplastics can even be eaten. ${ }^{14}$ Many papers ${ }^{15-17}$ define microplastics as particles below $5 \mathrm{~mm}$ in size and macroplastics for pieces large than $5 \mathrm{~mm}$.

This gives rise to the need to study the release produced by domestic washes, based on factors such as temperature, washing cycles, frequency of washing clothes, chemicals used, available programs, etc. ${ }^{17}$ In recent decades, several technologies and sewage treatment systems for wastewater have been developed. Most of them focus on the removal of contaminants present in the chemical processes of the textile transformation chain. There are several categories that classify these cleaning systems, according to the type of method, composition, characteristics and concentration of the material. ${ }^{18}$

One of these cleaning methods is the preliminary treatment that eliminates thick suspended materials, such as threads, fluff, fibers, etc. This is achieved through the use of bars, thin screens and mechanical scraping systems. Flotation is another of the existing systems for the removal of small fibers in wastewater. It is achieved through the effect of interfacial tension, buoyancy, hydrostatic pressure and the intervention of other forces, which ensure that the fiber remains on the surface and can be eliminated. ${ }^{18}$ Despite these systems, at present no specific cleaning protocol for wastewater from washes is applied, leaving these microplastics in effluents that are discharged into the seas and oceans.

Yang et al. ${ }^{17}$ reported that one garment can produce around 1900 microfibers and, furthermore, they studied the influence of soap, which can increase by up to $75 \%$ the microfiber release. They work under the understanding of microfibers as microplastics. To emphasize the importance, they remark that some publications stated that a country such as Norway can release between 100 and 600 tons of fibers from households.

Some studies attempted to determine the presence of microplastics from textiles, relating them to different parameters such as the age of clothes, ${ }^{19}$ microplastics from fleece ${ }^{20}$ or considering the composition. ${ }^{14}$ 
Thus, the main objective of this paper is focused on determining the influence of the weave pattern on the microplastic release during laundering. During laundering there are many factors that can enhance the number of microplastics. In this research we have focused mainly on the influence of the design of the fabric, without considering external parameters such as soap or abrasion on the washing machine. The hypothesis is that the weave pattern or the compactness of fibers can influence the release of fibers during laundering. The general objective of the present work is based on the release of fibers in household washes and the influence of fabric design focused on the interlacing coefficient (IC). The purpose of the work is to establish a relationship between the quantity of microplastics and the IC. This finding can help the textile industry, particularly weavers, to select a more sustainable weave rapport regarding the microplastic release.

\section{Experimental details}

\section{Materials}

The fabrics were comprised of a textured polyester warp of 16.7 tex with 48 filaments and with no twist (167 dtex f48 t0) and an acrylic staple yarn for weft of 64 tex with 880 twists per meter (64 tex Z 880). Regarding the fabric density, for the warp, its density is $60 \mathrm{ends} / \mathrm{cm}$ and with two weft densities of 10 and 15 picks $/ \mathrm{cm}$. They were weaved in a Smit GS 900 weaving machine of $190 \mathrm{~cm}$ width, with a Stäubli DX-100 electronic Jacquard machine. The mass per unit area for the fabric with 10 picks $/ \mathrm{cm}$ is $200 \mathrm{~g} / \mathrm{m}^{2}$ and for the fabric with 15 picks $/ \mathrm{cm}$ it is $240 \mathrm{~g} / \mathrm{m}^{2}$.

The weave patterns studied were plain, satin and twill. Satin and twill were studied with different rapports: for twill it was $4 \times 4$ and $5 \times 5$, whereas for satin it was $5 \times 5$ and $8 \times 8$, all of which included plain weaves with both 10 and 15 ends/cm. Despite the fact that plain weaves have some modifications, they are not considered plain at all. Hence, plain weaves have no possibility of variation, as there is only one option.

Interlacing warps and wefts according to the specific rapport offers different interlacing coefficients (ICs). The weave IC, according to the definition by Galcerá ${ }^{21}$ and others, ${ }^{22}$ has been calculated by the following equation

$$
I C=i / w 1 \cdot w 2
$$

where $i$ fits with the number of interlacing points in the weave pattern, $w 1$ is the number of ends in the weave pattern and $w 2$ is the number of picks in the weave pattern.
Table 1 shows the different references assigned to each sample and the IC for each sample, and the exact rapport.

\section{Methods}

\section{Laundering method}

The fabrics were washed according to the standard EN ISO 105-C06, Color fastness to domestic and commercial washing. The A1S test was conducted according to the parameters listed below and using the Linitest:

four test specimens of dimensions of $5.5 \mathrm{~cm} \times 5.5 \mathrm{~cm}$;

$40^{\circ} \mathrm{C}$;

10 stainless steel balls;

$150 \mathrm{~mL}$ distilled water;

30 minutes;

without adjusting $\mathrm{pH}$.

In order to avoid introducing new particles into the wastewater apart from those from the fabric, soap and chemicals were avoided.

\section{Filtering}

Once the fabrics had been treated, the wastewaters were collected and filtered with a filter pump system, using fiberglass filters with a pore size of $1.2 \mu \mathrm{m}$ and $47 \mathrm{~mm}$ diameter. The fiberglass filter, supplied by Akralab, S.L., was placed in a magnetic filtering funnel that ensured all the water was filtered, preventing water leaks.

The fiberglass filters had been previously dried in an oven at $105^{\circ} \mathrm{C}$ for $2 \mathrm{~h}$ in order to avoid the presence of moisture. Once the wastewater had been filtered, filters

Table I. Characteristics of fabrics

\begin{tabular}{|c|c|c|c|c|c|}
\hline Reference & $\begin{array}{l}\text { Weave } \\
\text { pattern }\end{array}$ & Rapport & Course & $\begin{array}{l}\text { Density } \\
\text { picks/ } \\
\mathrm{cm}\end{array}$ & $\begin{array}{l}\text { Interlacing } \\
\text { coefficient }\end{array}$ \\
\hline PIO & Plain & lel & $I \times I$ & 10 & I \\
\hline PI5 & & lel & $I \times I$ & 15 & 1 \\
\hline T4IO & Twill & $3 e l$ & $4 \times 4$ & 10 & 0.5 \\
\hline T510 & & $4 \mathrm{el}$ & $5 \times 5$ & 10 & 0.4 \\
\hline T4I5 & & $3 e l$ & $4 \times 4$ & 15 & 0.5 \\
\hline T5I5 & & $4 \mathrm{el}$ & $5 \times 5$ & 15 & 0.4 \\
\hline S5I0 & Satin & $3 e 2$ & $5 \times 5$ & 10 & 0.4 \\
\hline S8I0 & & $5 \mathrm{e} 3$ & $8 \times 8$ & 10 & 0.25 \\
\hline S5I5 & & $3 e 2$ & $5 \times 5$ & 15 & 0.4 \\
\hline S8I5 & & $5 e 3$ & $8 \times 8$ & 15 & 0.25 \\
\hline
\end{tabular}


with fibers were dried in an oven at $105^{\circ} \mathrm{C}$ for $4 \mathrm{~h}$ to ensure no moisture was present in the filter or in the fibers.

The quantity of fibers kept on the filter is determined by the gravimetric difference, calculated according to the following equation

$0 / 00=($ weight after filtering - weight before filtering $)$ /weight before filtering $\times 1000$

\section{Rubbing test}

Some samples were placed on the artifact for abrasion testing. The fabrics were rubbed for 15 mins against a wool abradant fabric according to the ISO standard. Figure 1 shows the appearance of a fabric before (Figure 1(a)) and after (Figure 1(b)) the abrasion test.

Sample observation. The fiberglass filter was observed with an Olympus SZ-CT microscope, with a Zazi camera and suitable software (SZSS2000). The observation of the samples was also carried out by means of scanning electron microscopy (SEM) with a field emission scanning electron microscope (FESEM; ULTRA 55, ZEISS). Each sample is placed on a surface and covered with a layer of gold and palladium in order to transform it to conductive by using a sputter coater and covering it with gold. The samples were analyzed with the appropriate magnification and with an acceleration voltage of $1 \mathrm{kV}$.

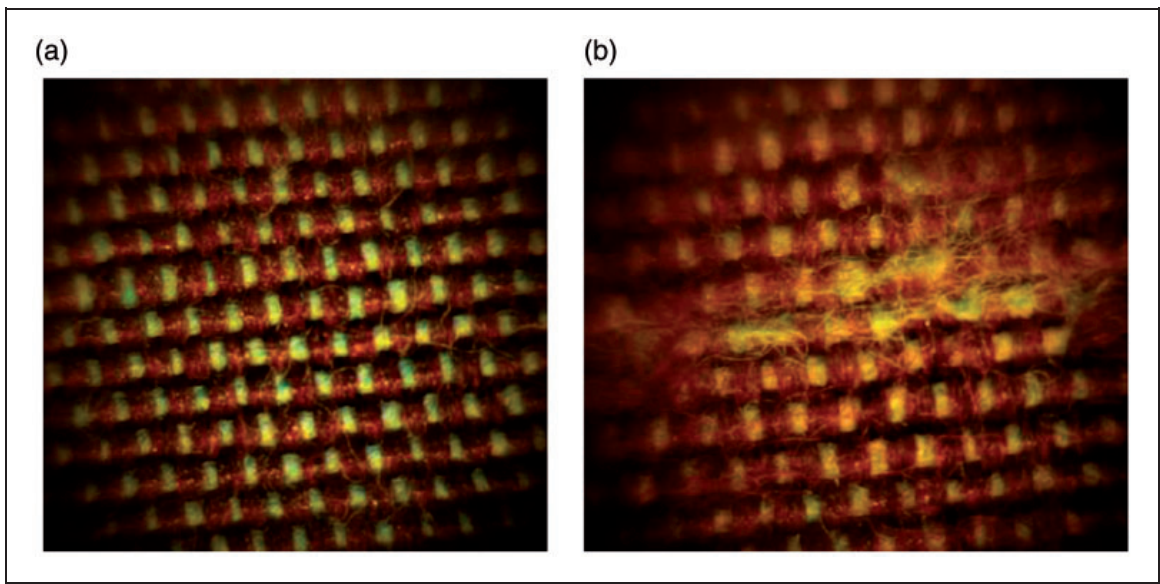

Figure I. Appearance of the fabric: (a) before rubbing test, magnification $2 \times$; (b) once the fabric has been rubbed, magnification $4 \times$.

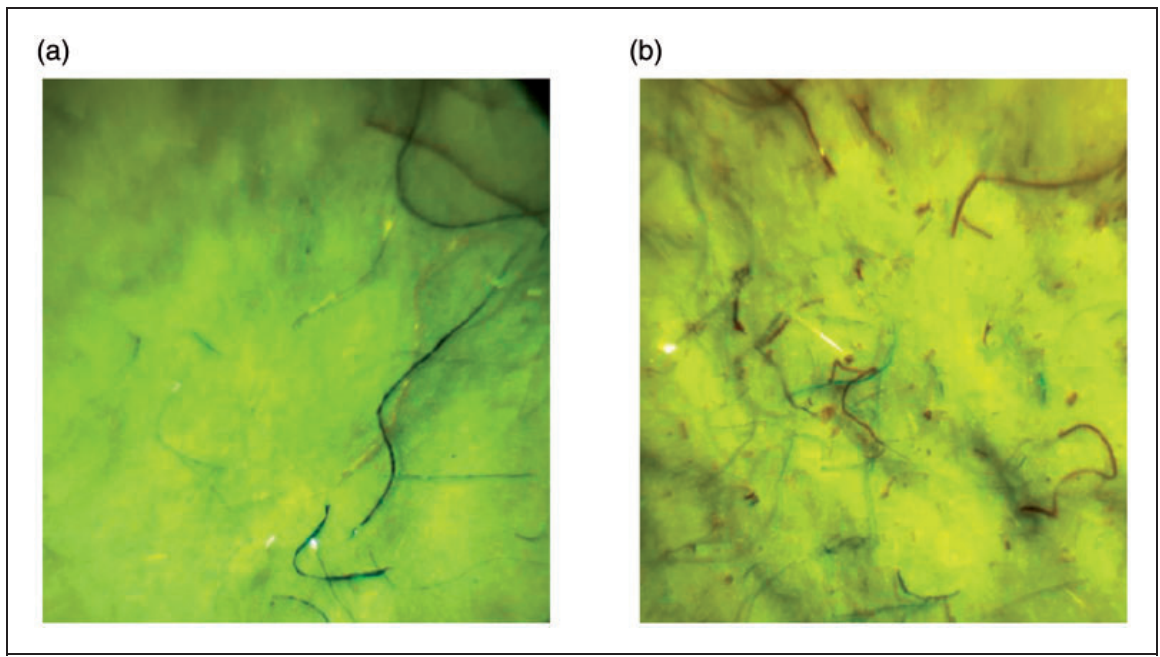

Figure 2. Appearance of the fiberglass filter, magnification $8.3 \times$ : (a) after filtering wastewater from a fabric; (b) after filtering wastewater from a rubbed fabric. 


\section{Results}

The first analysis was conducted by the observation of the filters in order to determine whether the fiberglass

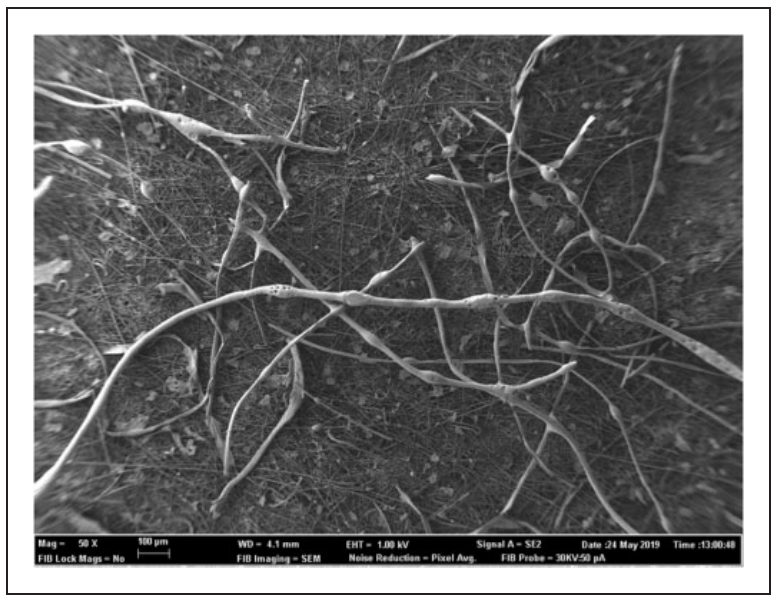

Figure 3. Field emission scanning electron microscope image of the fiberglass filter after filtering wastewater from a fabric.

Table 2. $\%$ of fibers in wastewater according to the weft density

\begin{tabular}{llllll}
\hline Density & P & T4 & T5 & S5 & S8 \\
\hline I0 picks/cm & 0.47 & 0.6649 & 0.635 & 0.5574 & 0.708 \\
SD & 0.054 & 0.0329 & 0.0296 & 0.0642 & 0.034 \\
I5 picks/cm & 0.2646 & 0.3688 & 0.327 I & 0.4385 & 0.6899 \\
SD & 0.0521 & 0.0468 & 0.0541 & 0.0358 & 0.0277 \\
\hline
\end{tabular}

filter was capable of retaining the fibers. Figure 2 shows some fibers on the fiberglass filter. It is clearly noticeable that once the fabric has been washed (Figure 1(a)) it shows some fibers on its surface, which seem to be longer than the fabric that has been rubbed previously in the laundering (Figure 1(b)). However, the fabric that had been previously rubbed, although shorter, seems to present more fibers in the filter (Figure 1(b)).

Considering that the fabric is comprised of polyester warp and acrylic weft, Figure 3 evidences not only the presence of fibers, which can be observed in Figure 2, but allows one to define see that the composition of fibers is synthetic, which come from the fabric.

When the study focuses on the rapport influence, three basic weave patterns are studied: plain, twill and satin. Three samples for each fabric were washed, and results show the average values from the three tests; the standard deviation is also offered (Table 2). Figure 4 shows the quantity of fibers in wastewater from fabrics with 10 picks $/ \mathrm{cm}$. The fabric seems to behave with three different tendencies: the plain fabric $(\mathrm{P})$ shows the lowest quantity of fibers, whereas the satin $8 \times 8$ (S8) fabric seems to wash out more fibers, and the remaining fabrics (T4, T5 and S5) show an intermediate behavior. However, when this behavior is compared with the weft density it can be clearly observed (Table 2) that both plain and twill practically double the $\% / 00$ of fibers in wastewaters, whereas satin shows practically the same values.

In order to determine the existence of a relationship between the quantity of fibers in wastewater and the IC, the results are represented in Figure 5. It is clearly

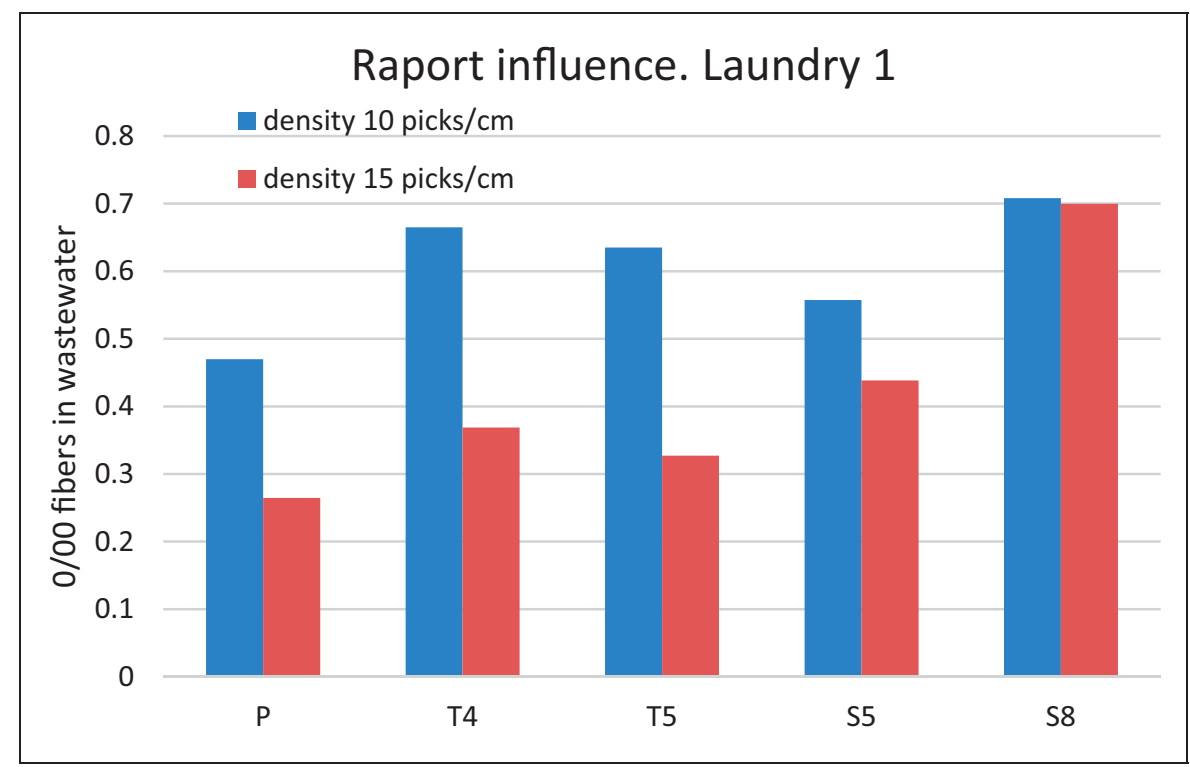

Figure 4. $\%$ of fibers in wastewater according to the weave pattern in the first laundering. 


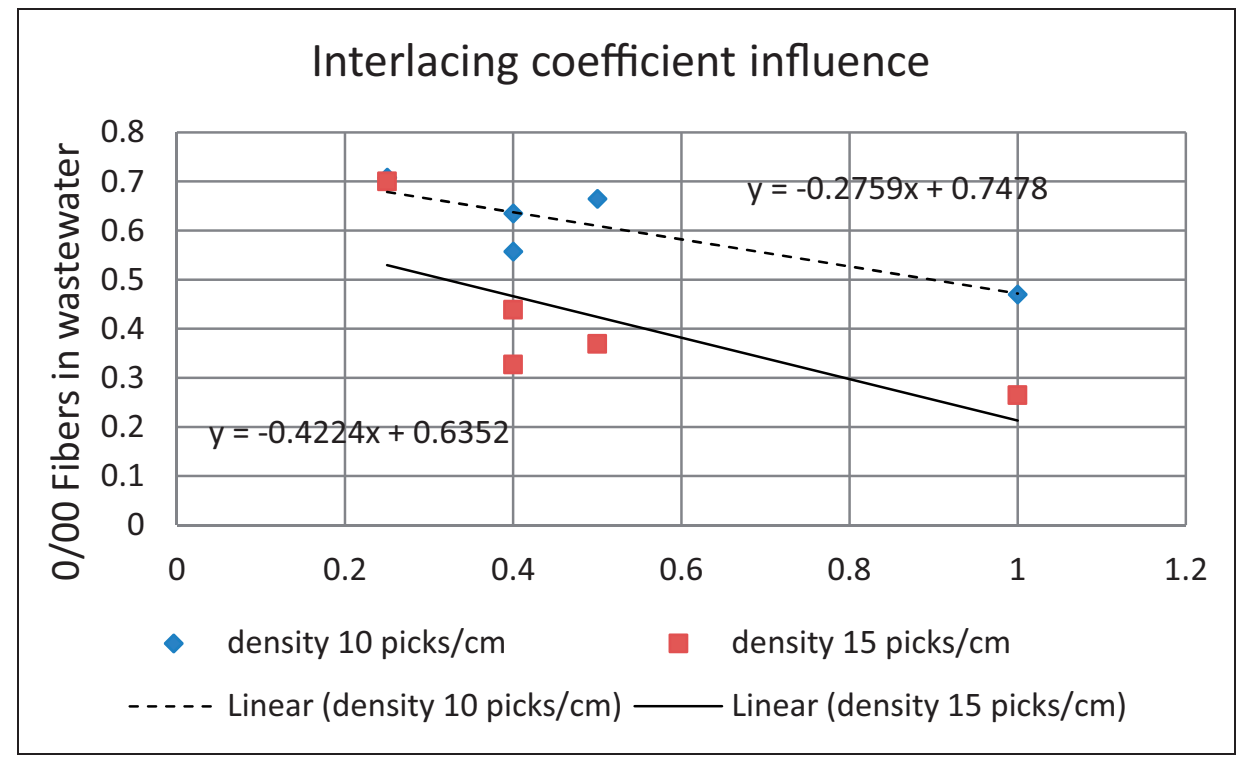

Figure 5. $\%$ of fibers in wastewater according to the interlacing coefficient.

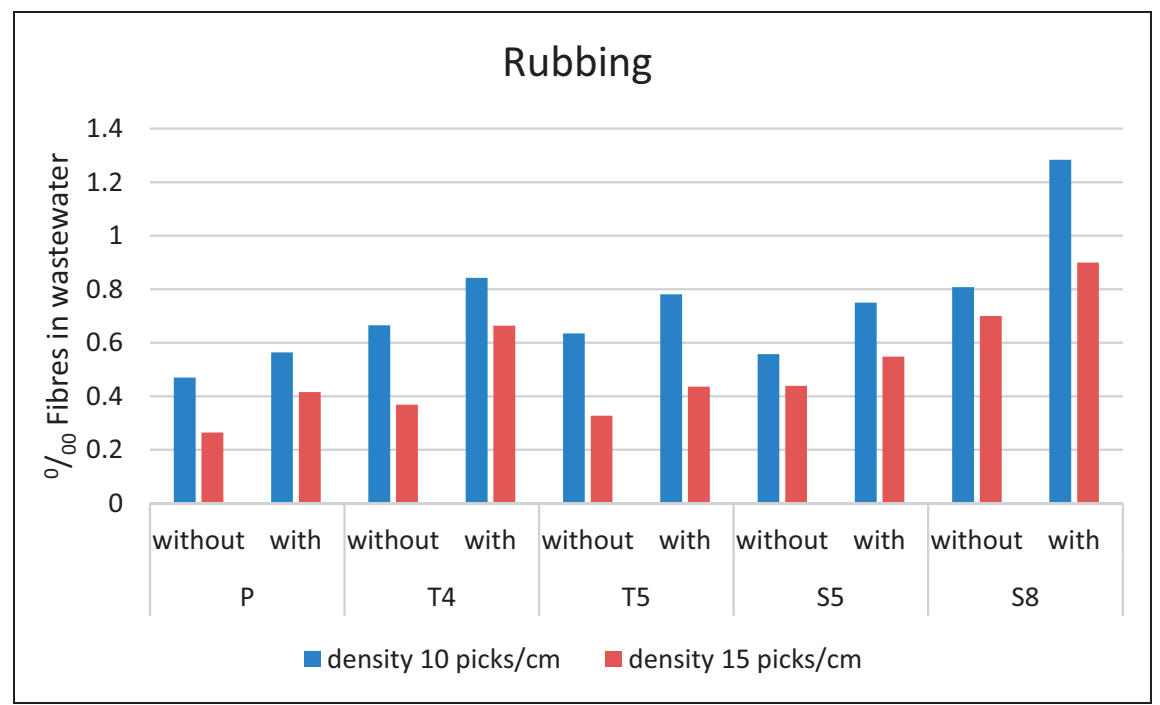

Figure 6. $\%$ of fibers in wastewater on the rubbed fabric.

observed that there is a decreasing tendency (line with negative slope). Considering both densities (10 and 15 picks $/ \mathrm{cm}$ ), the number of fibers is inversely proportional to the IC. When the density is increased it is supposed that we will observe a higher quantity of fibers as we include more yarns in a centimeter of fabric. To our surprise it is shown that with a higher weft density, the amount of fibers that will wash out from the fabric is lower. This can be observed by the lower value on the constant value in the equation ( 0.6532 for 15 picks $/ \mathrm{cm}$ and 0.7847 for 10 picks $/ \mathrm{cm}$ ). We consider this to be due to the fact that when the weft density is increased the fiber compactness is higher and, consequently, the fibers are tightened and their freedom to move toward the washing bath is not as high.

The results show that there is no any relationship between the number of microplastics released from the fabrics during the laundry and the weave pattern. So, the IC is suggested as a suitable parameter with which to establish a relationship with the number of fibers. High values for the IC imply the yarns are tighter and, consequently, the number of fibers migrating out of the yarn must be lower.

Thus, we can state than the higher the density, the lower the number of fibers in the wastewater, although 
by means of increasing the IC, the number of fibers can also be diminished. The reduction in the number of fibers in the wastewater can be attributed to the higher crisscross created by increasing the IC and the density, hindering the fiber migration from the fabric to the bath.

Figure 6 shows the number of fibers in the wastewater in comparison with the rubbed fabrics. It is clearly observed that there are more fibers in the wastewater in from fabrics rubbed previous to the laundry treatment. This effect is due to the mechanical action on the fabric surface during the rubbing action, which allows fibers to migrate to the fabric surface, as can be observed in Figure 1, and makes easier the migration of fibers toward the water during laundering.

The analysis from figure 6 shows not only that rubbed fabrics wash out more fibers, but also confirm the tendency that fabrics with a higher IC (P) miss fewer fibers than the fabrics with a lower IC (S8). Furthermore, the influence of density is corroborated

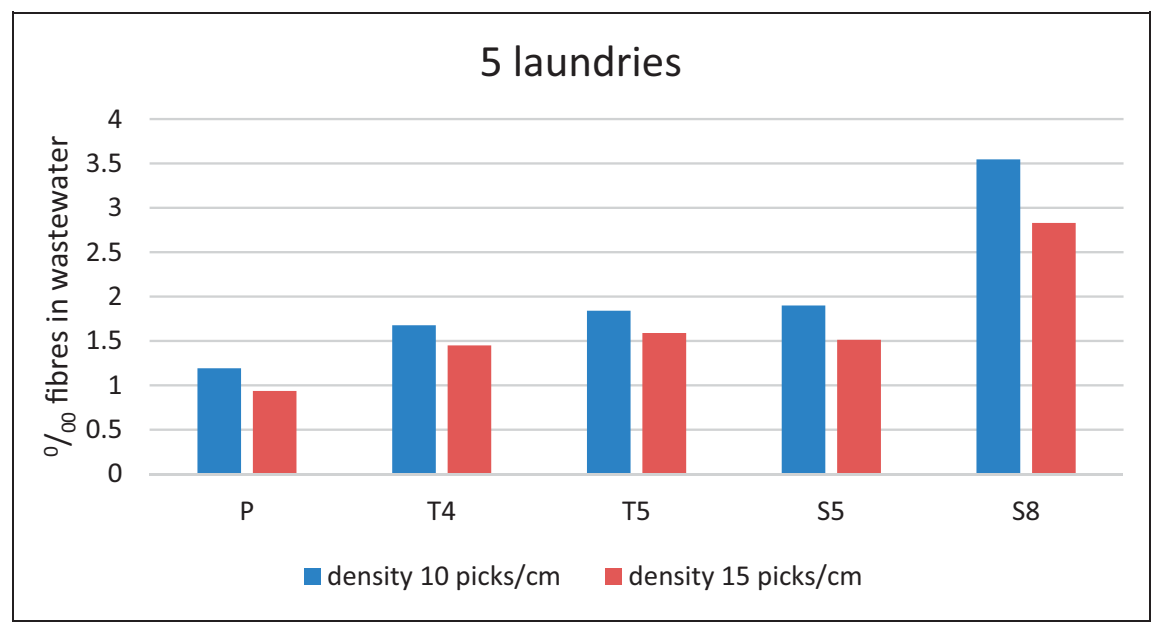

Figure 7. $\%$ of fibers in wastewater after five laundries.

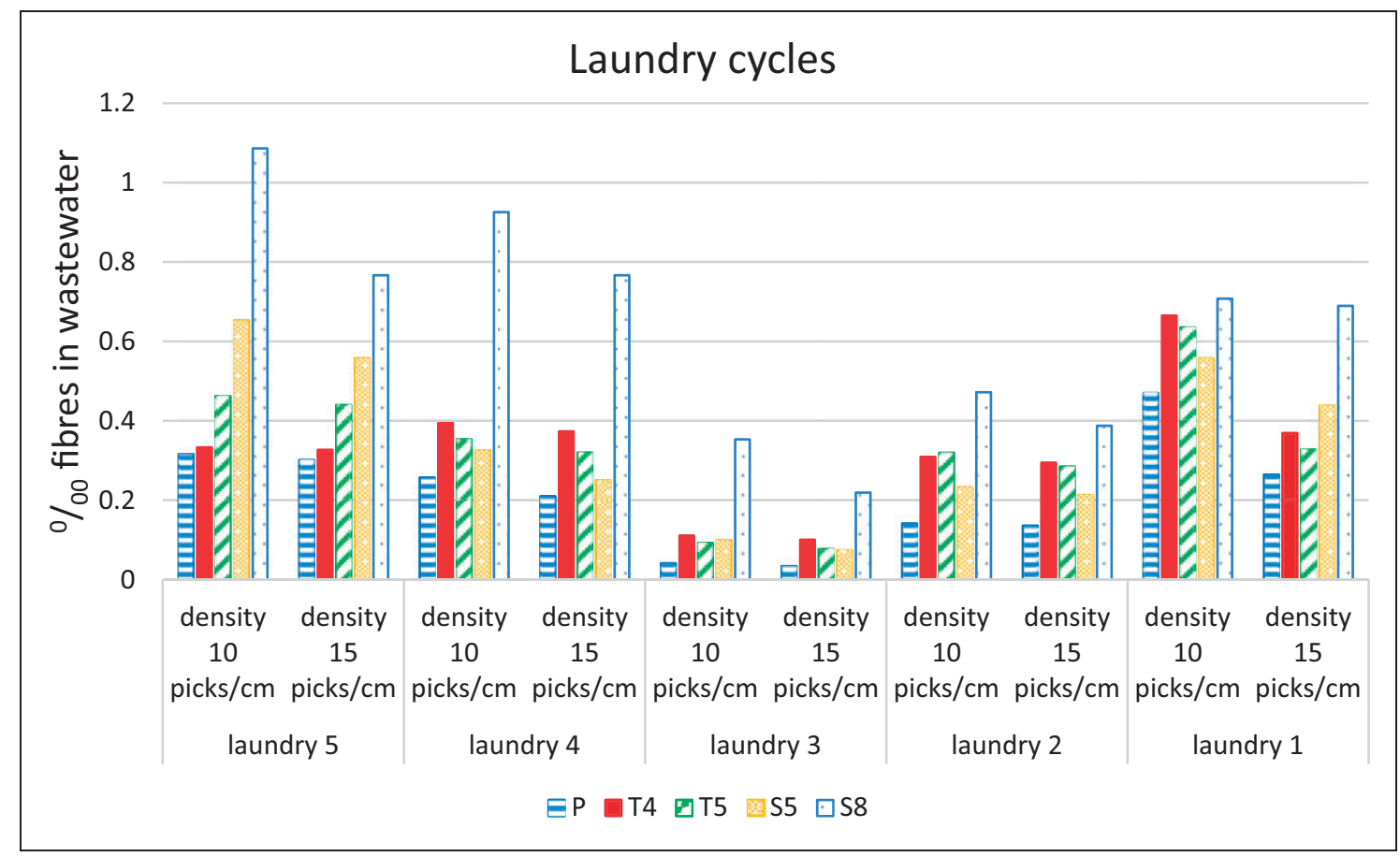

Figure 8. $\%$ of fibers in wastewater after five laundries. 
as the higher the density, the less the fibers escape from the fabric to the wastewater.

Regarding the study of successive laundries, five different laundry cycles were applied to the fabrics. From one laundry to the next one, the fabrics were flat dried at room temperature. Figure 7 offers a representation of the fibers in wastewater obtained by the addition of the fibers in wastewater from the first to the fifth laundry. Once more, we can state that the weave pattern has no influence on the release of microplastics and the tendency is perfectly related with the IC. When the accumulated total for the five laundries has been calculated, the highest IC shows the lower microplastic release and when the IC is the lowest, the release is the highest.

When the wastewater is analyzed cycle by cycle (Figure 8), the number of fibers is lower for cycles 2 and 3 but increasing cycle by cycle in the fourth and fifth cycles. It seems that the first washing releases the superficial fibers and, during the second and third washes, there are not so many fibers washed off, and it is after the fourth cycle when the number of fibers in the wastewater increases. Regarding the main focus of the article, the IC effect, Figure 7 shows that it is not altered by the number of cycles. Fabric S8 with the lowest IC is always the fabric with the highest amount of microplastics, whereas the plain fabric, with the highest IC, always shows the lowest amount. It is also interesting to note that T4, T5 and S5 with similar ICs reflect intermediate behavior, fitting with the intermediate IC.

Moreover, the weft density behavior is similar: the higher the weft density, the fewer microplastics can be found. Thus, when the IC is higher fewer fibers escape from the fabric.

\section{Conclusions}

Nowadays, textiles are considered to be a significant pollutant, partially due to the fibers present in wastewaters from domestic laundries. Fortunately, textile technology is extensive enough to minimize the environmental impact from the industrial process, although further studies should be conducted to determine some of the conditions necessary to develop or maintain sustainable textiles.

In this study the influence of the rapport and IC on the presence of fibers in wastewater from laundering has been studied. Three basic weave patterns have been studied: plain, twill and satin. It has been demonstrated that it is not the weave pattern but the IC that is most important aspect to consider, such that the higher the IC, the lower quantity of fibers would be moved from the fabric into the wastewater from laundering.
Despite what was expected, when the density of yarns is increased, the number of fibers in wastewater decreases. Inserting more yarns/cm implies more fibers; however, the number of fibers was reduced due to the fact that fiber compactness is higher and they do not move from the fabric easily. Furthermore, it has been demonstrated that the rubbing process improves the number of fibers in the wastewater. It has been demonstrated that the number of fibers increases in each laundering, at least until the fifth cycle.

To sum up, we can confirm there is a strong relationship between the design of the fabric and the release of fibers in laundering. The weave pattern is not a criteria for design, but the IC must be considered a suitable indicator. Hence, manufacturers, particularly weavers, who are deeply concerned with environmental issues must include, if possible due to design requirements, weave patterns with a higher IC and/or higher densities of yarns to increase the compactness of the fibers and prevent the release of fibers in wastewaters.

\section{Declaration of conflicting interests}

The authors declared no potential conflicts of interest with respect to the research, authorship, and/or publication of this article.

\section{Funding}

The authors received no financial support for the research, authorship and/or publication of this article.

\section{ORCID iDs}

Marilés Bonet-Aracil (D) https://orcid.org/0000-0002-8743560X

Pablo Díaz-García (D) https://orcid.org/0000-0002-7093-6061

\section{References}

1. Andrady AL. Microplastics in the marine environment. Marine Pollut Bull 2011; 62: 1596-1605.

2. Beckman E. The world's plastic problem in numbers. World Economic Forum, https://www.weforum.org/ agenda/2018/08./the-world-of-plastics-in-numbers (2018) (accessed 20 January 2019).

3. Crespy D, Bozonnet $\mathrm{M}$ and Meier M. 100 years of Bakelite, the material of a 1000 uses. Angewandte Chemie Int Ed 2008; 47: 3322-3328.

4. Napper IE and Thompson RC. Release of synthetic microplastic plastic fibres from domestic washing machines: effects of fabric type and washing conditions. Marine Pollut Bull 2016; 112: 39-45.

5. Korol J, Hejna A, Burchart-Korol D, et al. Water footprint assessment of selected polymers, polymer blends, composites, and biocomposites for industrial application. Polymers 2019; 11: 1791.

6. Gan L, Guo H, Xiao Z, et al. Dyeing and characterization of cellulose powder developed from waste cotton. Polymers 2019; 11: 1982. 
7. Koszewska M. Circular economy - challenges for the textile and clothing industry. Autex Res J 2018; 18: 337-347.

8. Lee MS, Seifert M and Cherrier H. Anti-consumption and governance in the global fashion industry: transparency is key. In: Hira A and Benson-Rea M (Eds), Governing corporate social responsibility in the apparel industry after Rana Plaza. New York: Macmillan, Palgrave, 2017, pp.147-174.

9. Yu F, Yang C, Zhu Z, et al. Adsorption behavior of organic pollutants and metals on micro/nanoplastics in the aquatic environment. Sci Tot Environ 2019; 694: 133643.

10. Cole M, Lindeque P, Fileman E, et al. Microplastic ingestion by zooplankton. Environ Sci Technol 2013; 47: 6646-6655.

11. National Geographic. https://www.nationalgeographic. es/medio-ambiente/2018/10/hallan-microplasticos-en-el90-por-ciento-de-la-sal-de-mesa (accessed 16 October 2020).

12. Prata JC. Microplastics in wastewater: state of the knowledge on sources, fate and solutions. Marine Pollut Bull 2018; 129: 262-265.

13. De Falco F, et al. Evaluation of microplastic release caused by textile washing processes of synthetic fabrics. Environ Pollut 2018; 236: 916-925.
14. Zambrano MC, Pawlak JJ, Daystar J, et al. Microfibers generated from the laundering of cotton, rayon and polyester based fabrics and their aquatic biodegradation. Marine Pollut Bull 2019; 142: 394-407.

15. Prata JC. Microplastics in wastewater: state of the knowledge on sources, fate and solutions. Marine Pollut Bull 2018; 129: 262-265.

16. Cole M, Lindeque P, Halsband C, et al. Microplastics as contaminants in the marine environment: a review. Marine Pollut Bull 2011; 62: 2588-2597.

17. Yang L, Qiao F, Lei K, et al. Microfiber release from different fabrics during washing. Environ Pollut 2019; 249: 136-143.

18. Mostafa M. Waste water treatment in textile Industriesthe concept and current removal technologies. $J$ Biodivers Environ Sci 2015; 7: 501-525.

19. Hartline NL, et al. Microfiber masses recovered from conventional machine washing of new or aged garments. Environ Sci Technol 2016; 50: 11532-11538.

20. Pirc U, et al. Emissions of microplastic fibers from microfiber fleece during domestic washing. Environ Sci Pollut Res 2016; 23: 22206-22211.

21. Galcerán V. Weaving technology. Terrassa: Technical University of Catalonia, 1962.

22. Özdemir H. Air permeability of worsted fabrics. Industria Textila 2018; 69: 322-327. 